\title{
A PROVA DO FRIO NO ESTUDO DA PRESSÃO ARTERIAL (*)
}

\author{
HELIO LOURENÇO DE OLIVEIRA \\ Médico interno voluntário da $2 .^{a}$ M. F. da Santa Casa, Serviço \\ do Prof. Rubião Meira.

\section{MERRAME ADURA - MATHEUS ROMEIRO NETO \\ Estudantes-internos voluntários}

A pressão arterial está sujeita a variar consideravelmente, em função das reações do seu sịstêma regulador a estímulos internos e externos. A escolha de um estímulo conveniente, aplicado de módo constante numa prova "standard", permitiria medir, pelo valor da variação da pressão sanguínea, o gráu de excitabilidade do aparelho vaso-motor.

Em 1932, foi uma tal prova proposta por Hines e Brown, tendo os referidos AA. relatado pela primeira vez a resposta da pressão sanguínea a esse estímulo "standard", o frio aplicado localmente sobre a pele, que eles demonstraram produzir efeitos vasopressores em 99\% dos indivíduos; a reação se denominou "cold pressor test" (C. P. T.).

TÉcnica: Segundo Hines e Brown (15) é a seguinte: o paciente permanece em posição supina, em um ambiente sossegado, durante 20 a 60 minutos. Fazem-se várias leituras da pressão até que esta atinja um nivel básico, isto é, que não apresente nenhuma variação apreciavel em tres ou quatro leituras sucessivas, intervalądas de alguns minutos. A mão oposta à do lado em que o aparelho de pressão é aplicado, é então mergulhada em agua com gelo $\left(1-4^{\circ} \mathrm{C}\right)$, até um nivel imediatamente superior ao punho. Fazem-se duas leituras da pressão, uma 30 e outra 60 segundos depois da imersão. Em seguida, retira-se a mão da agua gelada e toma-se a pressão de 2 em 2 minutos, até que volte ao nivel básico inicial.

Esta técnica, porém, não foi seguiida à risca pelos diferentes autores que investigaram sobre o valor do "cold pressor test" Assim é que Miller e Bruger (22) conservaram o paciente sentado, só

(*) O presente trabalho é uma ampliação da comunicação feita pelos AA. no 1.o Congresso dos Estudantes de Medicina em Sào Paulo. Neste, fazem eles uma revisão praticamente completa da bibliografia do assunto. 
fazendo a prova no leito, quando o paciente não pudesse deixá-lo. Esses mesmos AA. usaram um esfigmanômetro que não thes permitia medir a pressão 30 e 60 segundos após a imersão da mão em agua com gelo, e por isso tomavam duas vezes a pressão, tão depressa quanto possivel, evitando que a mão permanecesșe imersa -mais de 1 minuto: Schwab, Curb, Mathews e Schultze (26) procediam em tudo semelhantemente à técnica original, porếm faziam a mão permanecer imersa durante 150 segundos e faziam tres leituras: aos 30 , aos 60 e, finalmente, aos 150 segundos de imérsão.

Outros AA. procederam de módo a alterar màis ou menos a técnica original, o que motivou por parte de Hines JR. (19) uma advertência, em artigo onde descreve de novo a técnica e chama a atenção para diversos fatos, cujo conhecimento é necessario para a boa aplicação do "test" Em resumo, o referido A. diz: 20 minutos de repouso é tempo mais do que suficiente para um normotenso. $\mathrm{O}$ período de repouso necessario pàra um hipertenso é mais-longo, sendo , que quando a hipertensão é severa, a pressão șe estabiliza em nivel. alto e só se póde assegurar que estabilizou se ela permanecer aí por varias horas. $\mathrm{O}$ paciente deve permanecer deitado. Não sendo possivel isso, deve-se levar em contá o efeito da posição para a avaliação dos resultados: a pressão estabiliza-se em nivel mais alto quandó „o paciente permanece sentaido ou de pé, o que se dá particularmenter em relação à diastólica. Os efeitos hidrostáticos de sentar ou de ficar de pé farão diminuir a reação ao frịo, especialmente, se o paciente, fồ hipertenso.

$\mathrm{E}$ bom dizer ao paciente da- simplicidade da prova, no início do período de repouso, afim de evitar apreensões e facilitar a estabilização da pressão. 'A temperatura da agua não deve ir além de $4:^{\circ} \mathrm{C}$ e deve ser tomada antes de a mão mergulhar nela.

O paciente não deve tomar nenhuma droga sedativa ou vasomotora, nas vinte e quatro horas que precedem a prova. Drogas de eliminação lenta como oș brometos podem afetar os resultados em tempo superior a 24 horas. ',

Dados fornecidos pela prova - Uma vez estabilizada a pressão, mergulhando-se a mão na agua com gelo, observa-se que a pressão, tomada 30 e 60 segundos depois, não é igual à anterior. $\mathrm{A}^{\prime}$ máxima e a mínima, via de regra, acham-se aumentadas. Esse aumento varia com os casos, não havendo relação fixa entre o da sistólica e o da diastólica. (figs. $1,2,3$ ). Por muito grandes que tenham sido as elevạções da pressão, ultrapassando por vezes a capacidade do aparelho de medida, Hines e Brown (15) nunca verificaram um acidente à aplicação da prova em várias centenas de casos.

A resposta é a diferença entre os valores máximos alcançadois pelas pressões sistólica e diastólica durante a excitação e os valores básicos respectivos, correspondentes à̀ pressão estabilizada pelo repouso. 
Exemplo: pressão estabilizada em $130 \mathrm{~mm}$. Hg. (sistol.) e $70 \mathrm{~mm}$. Hg. (diastol.) ; valores máximos obtidos durante a excitação, respectivamente 150 e 80 . Então: resposta da sistólica = $20 \mathrm{~mm}$. de $\mathrm{Hg}$. e resposta da diastólica $=10 \mathrm{~mm}$. de $\mathrm{Hg}$. Esse dado' é 'o que os autores norte-americanos denominam "range" ou "response"

Outros dados que se devem anotar são: o tempo que demora a pressão para voltar ao nivel básico, desde que termina a excitação pelo frio; e o valor absoluto atingido pela pressão, o acme ("ceilling" de Hines e Brown). Há um acme sistólico e um diastólico.

A representação gráfica dos resultados permite a facil apreensấo desses resultados (figs. 1, 2, 3).

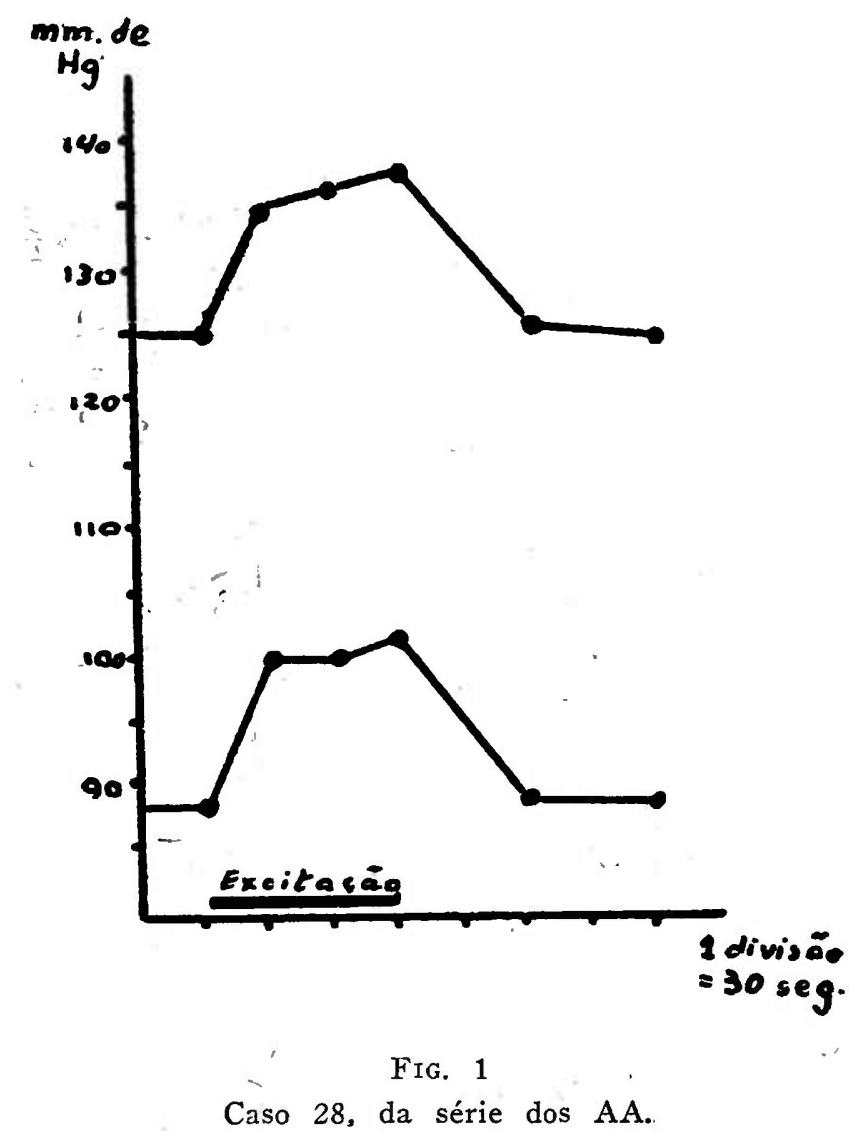

Mecanismo da reação - A explicação mais provavel, segundo Hines e Bown, é que a resposta à excitação corresponda a uma reação vaso-pressora generalizada, iniciada atravez de um arco reflexo nervoso. Segundo eles, não ha variação significativa da frequência e do débito cardíacos durante a prova. Em colegas nossos da Faculdade, aplicamos a prova de excitação pelo frio, controlando simultaneamente a variação da pressão e da frequência do pulso, ambas previamente estabilizadas; a pressão era medida aos 30, 60 e 90 segundos da excitação, e 1, 2 e 4 minutos após -a retirada do estímulo. O pulso era tomado entre o $300^{\circ}$ e $600^{\circ}$ segundo de excitação e mais duas vezes: antes do primeiro minuto e imediatamente depois do se- 
gundo minuto, após a retirada do estímulo. Em 25 casos assim controlados a frequênçia do pulso, ora aumentava, ora diminuia, ora permanecia inalterada, sem que se pudesse notar qualquer relação definida dessas variações com as respostas da pressão sanguínea.

Segundo aqueles AA., a adrenalina não é o fator da reação, em virtude da velocidade com que a reação se dá, da sua verificação em cães adrenalectomisados e da sua presença em pacientes com moléstia de Addison. A possibilidade de produção local de uma substância vaso-pressora, pela ação do frio, é tambem afastada pelos mesmos AA., pois que obtiveram resposta à prova impedindo, por meio-de um garrote, a circulação no membro cuja nião era imerśa na agua fria.

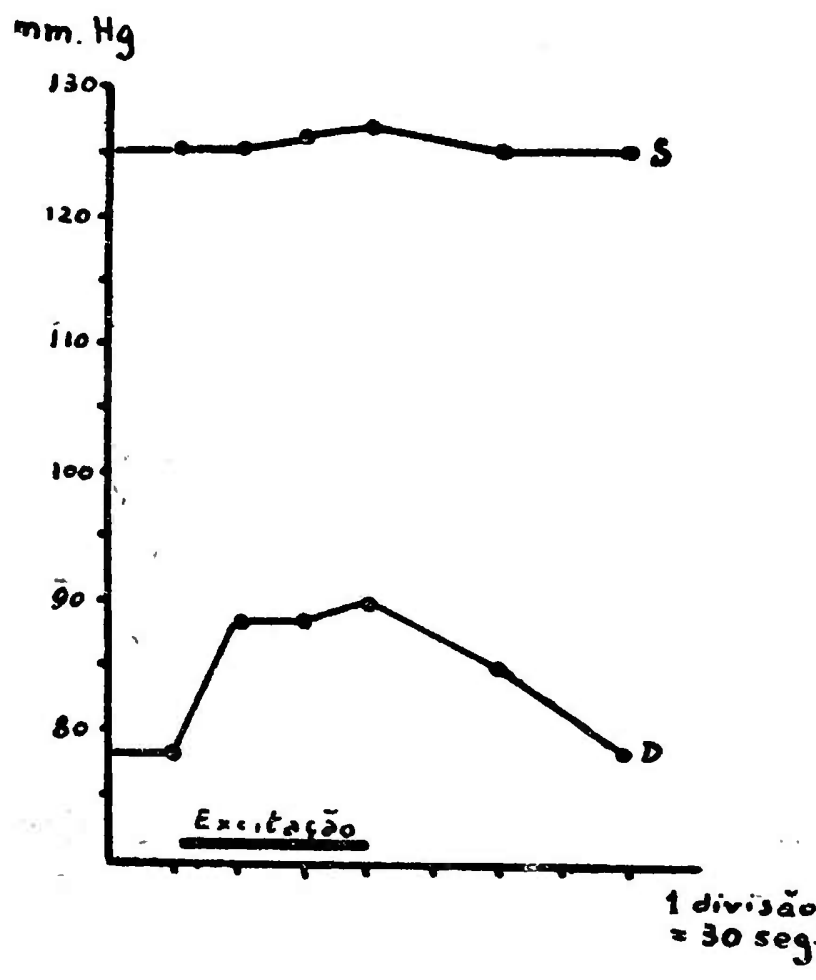

FIG. 2

Caso 3 , da série dos AA.

Tipos de reação - Classificação dos indivíduos segundo os mesmos. Os valores encontrados para as respostas ao "cold pressor test" variaram entre limites muito amplos, $e_{\theta}$ significativamente se verificou que os indivíduos com hipertensão essencial apresentavam respostas enquadradas entre as altas, enquanto indivíduos normo'tensos apresentavam de modo geral respostas mais baixas. Mas uma parte dos indivíduos com pressão normal exibiam respostas da mesma. ordem de grandeza das respostas dos hipertensos, em nada se distinguindo destes em relação ao "cold pressor test". Esse fato realmente é o centro do ìnteresse despertado pelo trabalho -de Hines e Brown, em torno de que giram as cogitações de ordem interpretativa e $\mathrm{em}$ relação ao qual se orientam todas as pesquizas -sobre o 
assunto; buscā-se estabelecer que relações positivas se podem admitir, ligando o grupo dos hipertensos ao grupo dos indivíduos normotensos que apresentam resposta elevada ao "test".

Caracterizam-se assim dois tipos de resposta à excitação pelo frio: o hiperreativo e o hiporreativo. Como o primeiro se verifica

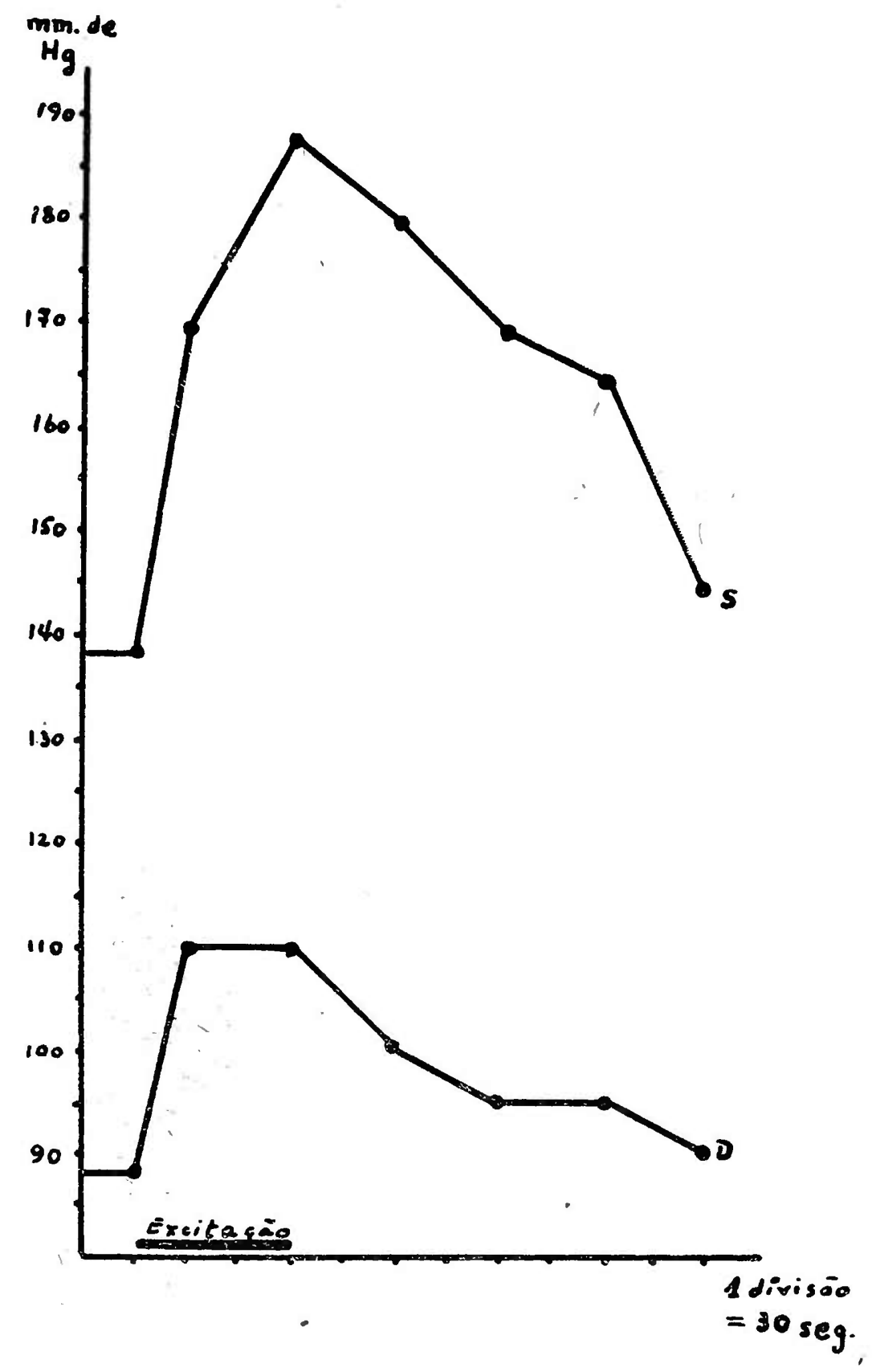

Fig. $;$

Caso 90, da série dos AA.

nos hipertensos eśsenciais, considera-se anormal, chamando-se de normal a resposta do tipo hiporreativo. A definição rigorosa desses dois tipos de reação evidentemente exige a determinação de um certo valor máximo abaixo do qual a resposta deva se considerar do tipo hiporreativo ou normal. Baseando-se em muitas centenas de provas 
em indivíduos hipertensos e normotensos, Hines Jr. (20) fixa em $22 \mathrm{~mm}$. de $\mathrm{Hg}$. o aumento da sistólica e $15 \mathrm{~mm}$. de $\mathrm{Hg}$. o da drastólica como valores maximos normais da resposta. (Esses limites foram fixados empiricamente e em trabalhos anteriorés não correspondiam a esses, que são contudo os baseados em estatísticas mais numerosas e adotados em trabalhos recentes). Pode haver hiperreatividade apenas na pressão sistólica ou na diastólica, porém para mais perfeita caracterização do tipo hiperreativo deve se exigir que ambos os limites sejam ultrapassados. (Fig. 3).

Os indivíduos, segundo sua pressão arterial e a resposta ao "test", se classificariam:

$$
\text { Normotensos }\left\{\begin{array}{l}
\text { hiporreativos } \\
\text { hiperreativos }
\end{array}\right.
$$

Hipertensos essenciais ${ }^{\top}$ - geralmente hiperreativos.

Nesse quadro, em que de propósito se omitem outros tipos de hipertensão, toma-se como limite de pressão arterial normal $140 \mathrm{~mm}$. de $\mathrm{Hg}$. para a sistólica e $90 \mathrm{~mm}$. de $\mathrm{Hg}$. para a diastólica.

O acme dos normais, hiporreativos é em $98,4 \%$ dos casos inferior a $145 \mathrm{~mm}$. na sistólica e em $99 \%$, inferior a $95 \mathrm{~mm}$. na diastólica, segundo a estatística de Hines e Brown (15); e $72 \%$ dos normais hiperreativos apresentam acmes superiores a 145-95. De acordo com os AA. reféridos, levar em conta apenas os acmes, ou apenas a resposta, seria arbitrário. Sấo de opinião que se um indivíduo, além da resposta excessiva, apresenta acmes excessivos (acima de 145-95), com mais fundamento se póde falar em um sistema vasomotor hiperreativo.

\section{SIGNIFICAÇÃO DOS RESULTADOS}

O tipo hiperreativo de resposta foi tomado por Hines e Brown. (15) como próprio de uma constituição hipertensiva, sendo que provavelmente todos os hipertensos foram anteriormente indivíduos normotensos hiperreativos. Fazendo cuidadoso inquérito sobre antecedentes familiares de moléstia cardiovascular hipertensiva entre indivíduos que apresentavam a pressão normal, aqueles AA. encontraram: de 90 hiperreativos, 78 apresentavam essa história positiva; e de 288 hiporreativos, apenas $14 \%$ com os antecedentes positivos. Acompanhando a evolução de 8 normotensos hiperreativos, registrados em 1932, os mesmos AA. registraram, em 1936, o aparecimento de graus clínicos de hipertensão em três deles (aumento da pressão sanguínea, e transformações características das arteríolas da retina). Já em 1939, Hines JR. (20) registra o desenvolvimento de hipertensão em $38 \%$ de 29 indivíduos antes normotensos hiperreativos, num período de 6 anos, durante o qual nenhum dos hiporreativos estudados se tornou hipertenso. 
Tais fatos sugeriram que os indivíduos normotensos com reação anormal ao "test" representam hipertensos em estado potencial; a hipertensão seria condicionada por um fator constitucional primário, manifestando-se por um sistema vaso-motor hiperreativo, ativado por fatores secundários, de natureza infecciosa, tóxica ou representado por excitações exógenas. Conceito que vem em apoio de uma teoria neurogênica da hipertensão.

Constância da reação - Verificações de outra ordem vieram em favor de tal modo de entender a significação das reações exageradas ao "test" A resposta à prova se apresenta como uma constante individual, muito aproximadamente; Hines e Brown (15), repetindo com intervalos variaveis de 3 mezes a 3 anos, o "cold pressor test" em 25 normotensos e 20 hipertensos, não encontraram variação apreciavel da resposta em 90\% desses indivíduos; nenhum dos que apresentavam anteriormente resposta igual a $18 \mathrm{~mm}$. de $\mathrm{Hg}$. ou in-

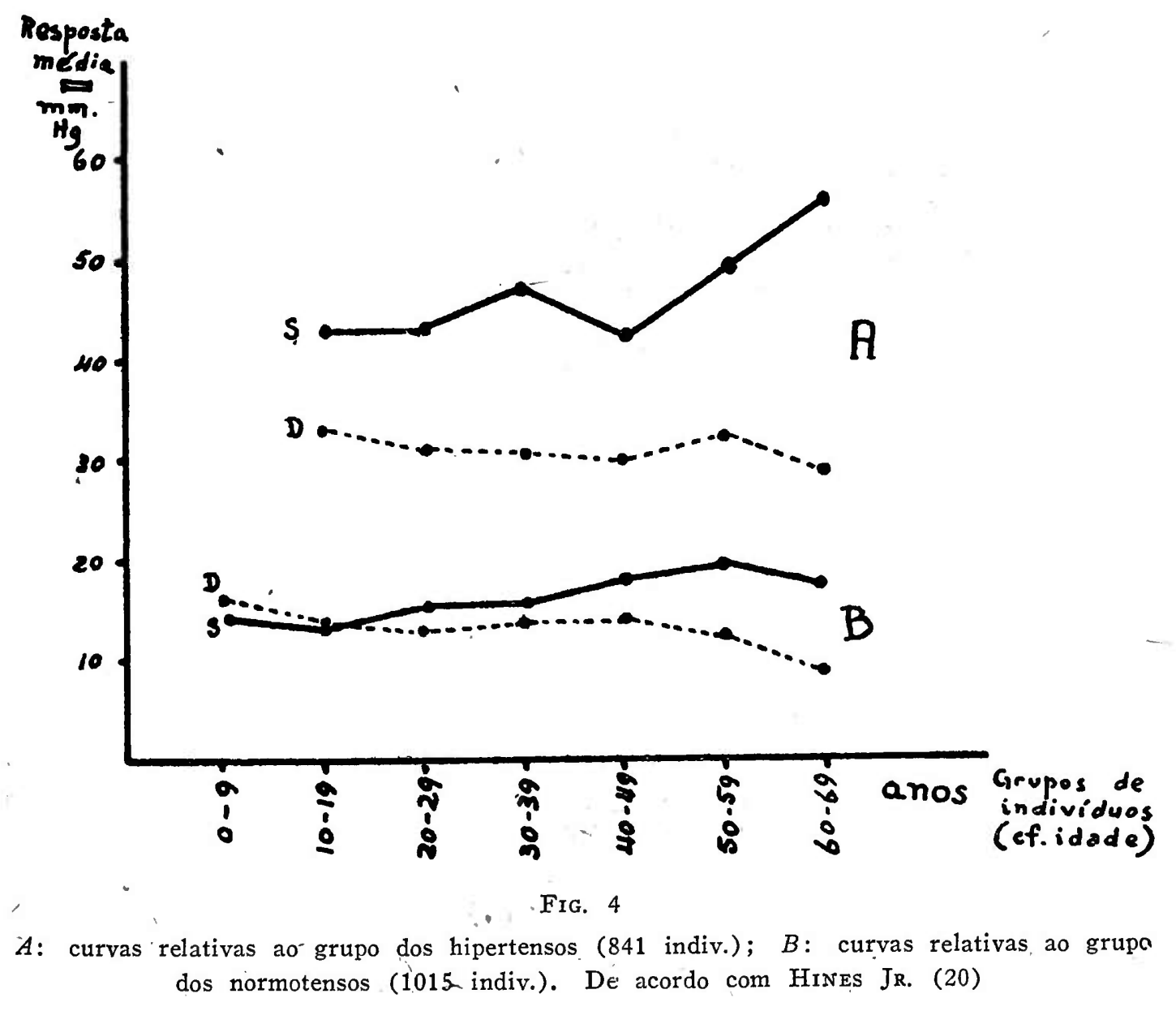

ferior, passou a dar respostas anormais (isto é, superior a $22 \mathrm{~mm}$. de $\mathrm{Hg}$ ): - Apenas alguns que forneceram respostas nos limites máximos normais deram ocasionalmente respostas nos mínimos anormais; são os AA. levados a admitir então um pequeno grupo indeterminado quanto ao tipo de reação. Indivíduos com hipertensão deram sempre respostas anormais às provas repetidas com intervalos 
de mezes ou anos. No Johns Hopkins Hospital, repetindo-se a prova de 5 a 10 vezes em 5 hipertensos, com intervalos de 2 a 10 dias, obtiveram-se sempre respostas hiperreativas (28):- HINES JR. (16) repetiu a prová em 40 crianças, após tres mezes de intervalo e não notou qualquer modificação significativa em relaçãa às primeiras provas; o mesmo A. reconhece que pode haver alguma variação da resposta em provas repetidas, que será mínima desde que se reproduzam sempre as mesmas condições basais e de realisação da prova. A constância da reação fica em todo caso subordinada não apenaș ao fator indivíduo mas, dentro de certos limites, ao fator idade, como levam a admitir os resultados de estudos estatísticos realizados sobre grupos de indivíduos normais e hipertensos de diferentes idades; isso vale sobretudo para a resposta sistólica: (ver gráfico; fig. 4). Porém, para Hines JR. o fato seguinte é significativo: embora haja um aumento definido na resposta médía com a idade, não ha nenhuma variação significativa na incidência de hiperreativos nos diferentes grupos de idade.

Hereditariedade do tipo de reação - Por outro lado, são interessantes do ponto de vista de tomar o tipo de reação à prová do frio como definidamente característico de uma "constituição vasomotora" individual, e muito favoraveis a essa idéa, os estudos sobre a herança do tipo de reação vaso-motora. São bem conclusivos os résultados do trabalho de Hines Jr. (17), de que traduzimos o resumo de que dispomos: "Foram orientadas observações em tres di-. reções: 1) correlação do tipo de reação ao "test" e da incidência de moléstia hipertensiva na história familiar; 2) estudo da reaçáa de gêmeoś ao "test"; 3 ) estudo da reação em membros de famílias. de hipertensọs e de não hipertensos. 1) Un estudo da história familiar de 608 pessoas que tinham pressão sanguínea normal e de 267 casos de hipertensão essencial mostrou que uma história familiar positiva de moléstia cardiovascular hipertensiva é 5 vezes. mais frequente entre os indivíduos que teem hipertensão ou são hiperreativos ao "cold test" do que entre os indivíduos que reagem normalmente ao "test". 2) 10 séries de gêmeos foram estudadas. Nos 7 grupos: de gêmeos idênticos a resposta de um foi idêntica à do outro, enquanto em 2 dos 3 grupos de gêmeos fraternos as respostas foram diferentes. 3) Foram estudadas 30 famílias, constituidás por 245 membros. Em 12 famílias não havia evidência ou hístória de moléstia cardiovascular hipertensiva; em 18 havia uma diátese hipertensiviva definida. Foram submetidos à prova todos os membros vivos. Quando ambos os pais tinham pressão sanguínea normal e eram hiporreativos, todos os filhos eram hiporreativos. Si ambos os pais eram hiperreativos ou tinham hipertensão, 95\% dos filhos reagiam do mesmo modo; e si um dos pais tinha hipertensão ou era hiperreativo e o outro era hiporreativo, 43,4\% dos filhos eram hiperreativos ou tinham hipertensão. Em 3 famílias de 2 gerações, os pais tinham hipertensão 
essencial ou haviam morrido dela, e todos os filhos tinham hipertensão essencial ou eram hiperreativos"

E evidente a importância de tais fatos para a avaliação do interesse que tem uma resposta de tipo hiperreativo ao "test" do frio; pais ambos hipertensos ou hiperreativos, transmitem a todos os filhos determinados fatores que lhes conferem ao sistema vaso-motor $o$ carater de hiperreatividade; todos os filhos se apresentam hiperreativos à prova do frio, ainda que em dado momento apenas alguns exibam hipertensão. Tais fatos são demonstrativos de que o que se transmite hereditariamente é un sistema vasomotor hiperreativo, permitindo sem dúvida uma nova compreensão do problema da hereditariedade da hipertensão.

Em resumo - O gráu de reatividade vasomotora é um elemento constante da constituição individual; o tipo de reação vasomotora, determinado pelo método do "cold pressor test", está definidamente ligado a fatores hereditários; a hipertensão essencial só se desenvolve sobre constituição vasomotora hiperreativa, e seu carater familiar é função do carater hereditário de tal constituição. Como nem todo hiperreativo vai à hipertensão, estabelecida, o problema da hipertensão essencial seria sobretudo a determinação dos fatores que impedem ou retardam o seu estabelecimento em indivíduos hiperreativos.

Considerando o fato de passar progressivamente à classe dos hipertensos uma parte dos normotensos hiperreativos, pode-se esperar que nos grupos de idades mais avançadas a proporção de respostas exageradas ao "test", na classe dos normotensos, seja progressivamente menor. Yates e Wood (29) aplicaram a prova do frio em 205 indivíduos de 8 a 70 anos de idade, buscando a verificação dessa consequência, como contra-prova da teoria de Hines e Brown; esses AA. julgam que os resultados obtidos são sugestivos, ainda que não à evidência, da possibilidade de a hipertensão se desenvolver indiferentemente nos hiperreativos e nos hiporreativos. Realmente os dados de YÁTes e Wood não são nada conclusivos, como se pode verificar do quadro abaixo, tirado do trabalho desses AA.:

\begin{tabular}{|c|c|c|c|c|c|c|}
\hline Idade & \multicolumn{2}{|c|}{$\begin{array}{c}\text { Abaixo } \\
\text { de } 30\end{array}$} & $\begin{array}{c}\text { Acima } \\
\text { de 30 }\end{array}$ & \multicolumn{2}{|c|}{ Abaixo } & \multicolumn{2}{|c|}{ Acima } & \multicolumn{2}{|c|}{ Abaixo } & \multicolumn{2}{|c|}{ Acima } \\
de 40 & de 50 & de 50 \\
\hline N. & 135 & 70 & 171 & 34 & 192 & 13 \\
\hline $\begin{array}{l}\text { Resposta sistó- } \\
\text { lica acima de 22 } \\
\text { mm. Hg. (\%) }\end{array}$ & 14,8 & 24,3 & 18,1 & 17,6 & 18,2 & 15,4 \\
\hline
\end{tabular}




\section{APLICAÇôES DO C.P. T."}

Estado pre-hipertensivo - O "cold pressor test", como ficou explícito nas considerações anteriores, permitiria a discriminação, entre os indivíduos normotensos, daqueles em que se poderá desenvolver uma hipertensão essencial. Estes, os normotensos hiperreativos, se encontrariam num "estado pre-hipertensivo". Será importante o seu conhecimento para a profilaxia da hipertensão, pois a observação mostra que nem todo hiperreativo se apresenta hipertenso na idade adulta; fatores secundários, que ainda estão por se determinar precisamente, são indispensaveis para que sobre o."estado pre-hipertensivo" se desenvolva à hipertensão no sentido clínico atual.

Hipertensão latente - A hiperreatividade ao C. P T. permitirá em certos casos ligar a uma "hipertensão latente" lesões explicaveis por uma hipertensão arterial, porém _ presentes em indivíduo normotenso.

1) Hines JR. (20) observou mais de 100 indivíduos nas últimas décadas da vida, com pressão normal, sem história de hipertensão, muitos deles tendo verificado anos antes ter pressão normal, e que se apresentavam com alterações de arteríolas da retina próprias da hipertensão. Todos esses indivíduos deram respostas exageradas. ao C. P. T.. Aquele autor admite que essa hiperreatividade seja responsavel pelas alterações arteriolares, representando em- tais casos uma hipertensão latente.

2) Schwab e Curb (27) propuzeram a aplicação do C. P. T. para o diagnóstico de cardiopatia hipertensiva em cardíacos geral: mente de idade mediana, com grande aumento do coração, com ausência de lesões valvulares e pressão baixa: Ao lado das outras possibilidades, ha a de se tratar em tais casos de hipertensão arterial;", com abaixamento da pressão consequente à insuficiência cardíaca. A hiperreatividade ao C. P. T. se prestaria a atestar nesses casos a constituição hipertensiva, autorisando assim a se ligarem as alterações cardíacas a provavel hipertensão anterior, atualmente latente. Schawb e CuRb aplicaram a prova em 7 hipertensos que acompanhavam ha 3-10 anos e nos quais a pressão veiu a cair por insuficiência congestiva ( 5 casos), enfarto do miocárdio e infecção estreptocócica. Em 5 a resposta foi então entre 50 e $90 \mathrm{~mm}$. $\mathrm{Hg}$; noutro foi "limítrofe" e num só normal.

Hipertensão essencial e hipertensão de origem renal - Para distinção entre esses 2 tipos de hipertensão apresenta-se o C. P. T. como recurso de relativo valôr.

Alan e Smirk (1) e Miller e Bruger (22) aplicando o "test" em indivíduos normais, com hipertensão essencial e com hiper- 
tensão renal, chegaram a conclusões concordantes: nos casos de hipertensão de causa renal a resposta ao "test" é comparavel à dos normotensos hiporreativos. Para os primeiros AA. citados, o aumento da pressão diastólica e da sistólica nos casos de hipertensão renal foi menor que nos casos normais; para os últimos, foi levemente maior. Concordam ainda os dois trabalhos na verificação de respostas baixas em uma certa proporção dos hipertensos essenciais; no grupo de hipertensos essenciais estudados por Miller e BrUGER, reações exageradas ocorreram apenas em 76\% dos casos.

Pode-se concluir desses trabalhos: num indivíduo com pressão arterial elevada, a resposta exagerada ao C. P. T. afasta a possibilidade de hipertensão renal; a resposta baixa, ainda que indique probabilidade maior de não se tratar de hipertensão éssencial, não é decisiva.

Thomas e Warthin (28) estudaram a influência do frio sobre a pressão arterial de cães, trabalhando com animais normais e com animais previamente submetidos à constricção das arterias renais, segundo o método de GoldBLATt, nos quais se desenvolvera acentuada hipertensão. As respostas à excitação pelo frio não apresentaram, nesses dois grupos, diferenças definidas quanto aos valores médios e quanto à frequência de valores altos.

$\mathrm{Na}$ gravidez a aplicação do "cold test" de Hines e Brown, basea-se na -íntima ligação que existe, segundo certos autores, entre a hipertensão essencial e a toxemia gravídica (exclusive verdadeira nefrite). O "test" evidenciando os futuros hipertensos ou hipertensos potenciais, foi proposto para descobrir as pacientes em que provavelmente se desenvolverá a toxemia da gravidez.

Não ha concordância entre os diversos autores sobre o limite máximo da "resposta normal ao "cold test" em mulheres grávidas; Randal, Murray e Mussey e Reid e Teel consideram o limite máximo dessa resposta como sendo de $20 \mathrm{~mm}$. de $\mathrm{Hg}$. na pressão sistólica. Para Chesley e Chesley esse ralor é de $24 \mathrm{~mm}$. de Hg. DiECKMANN, Michel e WuDdoff admitem como normal uma elevação da pressão sistólica até $29 \mathrm{~mm}$. de $\mathrm{Hg}$.

Segundo alguns AA. o "cold test" é elemento valioso para prever-se uma futura toxemia gravídica; segundo outros, não se pode emprestar ao test esse valor.

No primeiro grupo temos Randall, Murray e Mussey (23) que encontraram $33 \%$ de toxemia nas hiperreativas e $0 \%$ nas hiporreativas; Briggs e Oerting (citados por Chesley e ChesLEY (7)) que nas hiporreativas observaram $0,9 \%$ de toxemia e nas hiperreativas, 48\%; Dieckmann, Michel e Woodruff (11) que observaram $11,3 \%$ de toxemia nas hiporreativas e $31 \%$ nas hiperreativas; 'BAK (4) que verificou uma resposta mais exagerada ao "cold test" nas pacientes que tivèram a toxemia gravídica e naquelas que tínham tendência a ter essa toxemia. 
No grupo dos autores que não emprestam valor à prova no sentido de que possa indicar iminência ou probabilidade da toxemia gravídica, temos: ReId e Teel (25) que encontraram 14,3\% de toxemia nas hiporreativas te $0 \%$ nas fiperreativas; CHesLey e Chesley (7) que obtiveram $10,3 \%$ de toxemia nas hiporreativas e $14,3 \%$ nas hiperrreativas.

A discordância entre os diversos autores pode ser explicada por várias causas:

1) Número pequeno de observações: Chesley e Chesley chamaram a atenção para esse fator: num grupo de 896 hiporreativas. às respostas sistólicas eles obtiveram 10,3\% de toxemia-e num grupo de 131 hiperreativas, a percentagem de toxemia foi de 14,3\%. Entretanto, nas-93 primeiras pacientes o "test" parecia provar bem: 12 hiperreativas e entre essas 5 casos de toxemia; nas 153 seguintes todas as toxemias foram em hiporreativas. Se as 2 séries, fossem tomadas separadamente teriamos conclusões opostas.

2) Não haver uniformidade quanto ao período da gravidez em que se deva aplicar o "test": REID e TEEL observaram variações das respostas nos diversos períodos da gravidez.

Chesley e Chesley aplicando o "test" no $10^{\circ}$ ou $3 .^{\circ}$ mês e no $8 .^{\circ}$ ou começo do $90^{\circ}$ mês da gravidez obtiveram diferenças nas respostas de 0/0 (sist./diast.) até 26/30, em média 5,8/7,0 $\mathrm{mm}$. Hg.

3) Não distinguir-se a toxemia de origem hipertensiva (de hipertensão essencial) da de origem nefrítica e principalmente nos casos de glomérulo-nefrite crônica.

No ciclo menstrual - Em trabalho recente, o italiano Croci (9) registra os resultados originais obtidos pelo método do C. P. T. aplicado diariamente durante todo o ciclo mesntrual. Em 14 mulheres perfeitamente sadias, nulíparas, entre 18 e $36^{\prime}$ anos, normais do ponto de vista ginecológico, com período menstrual de

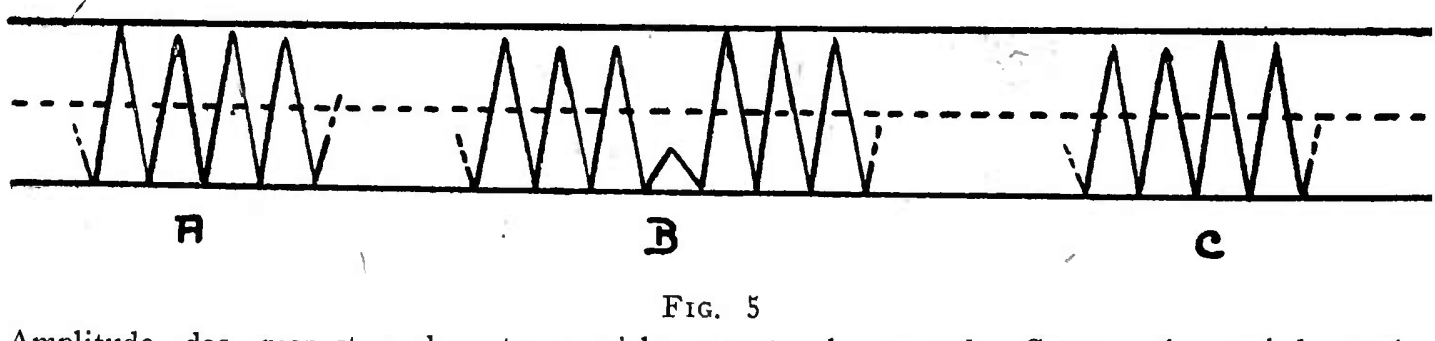

Amplitude das respostas durante o ciclo menstrual, segundo Crocr. $A$ : periodo postinenstrual. $B$ : p. intermenstrual. $C$ : p. premenstrual.

26 a 31 dias, encontrou resposta ao "test" aproximadamente constante durante todo o ciclo, a não ser em um dia do período intermenstrual, em que se verificava uma reação acentuadamente diminuida (v. fig. 5). Tal fáto foi verifícado do $110^{\circ}$ ao $19 .^{\circ}$ dia do ciclo menstrual. Crocr levanta uma hipótese: èssa diminuição da reatividade vasomotora corresponde à ovulação. 


\section{PROVAS EQUIVALENTES}

Outras classes de excitantes foram estudadas quanto à sua ação sobre a pressão arterial, além do frio da prova de Hines e Brown.

O exercício muscular do antebraço, coim parada da circulação, foi experimentado por Alan e SMIRK (1), que verificaram ter efeito vaso-pressor comparavel ao do frio aplicado segundo a técnica de Hines e Brow.

A parada voluntária da respiração ("breath-holding test") foi estudada por Ayman e Goldshine (2); verificaram de modo geral efeitos em tudo comparaveis aos do "cold test", e ainda com elevações mais acentua das da pressão.

Hines JR. e Roth (18) substituiram na prova primitiva a excitação pelo frio pelo ato de fumar 2 cigarros, e verificaram aumento da pressão mais acentuado nos indivíduos hipertenśos do que nos normais. Os hipertensos que deram respostas baixas eram não-fumantes, que provavelmente não tragavam a fumaça. Esses resultados talvez deem, si convenientemente verificados, uma primeira indicação no sentido da profilaxia da hipertensão em indivíduos hiperreativos.

Enfim, Hines Jr. (21) estudou a influência da excitação psíquica sobre a pressão, estudando 1522 pácientes que tinham vindo à consulta pela primeira vez 10 e 20 annos antes, e que voltaram à consulta após esse tempo; considerou os valores da pressão tomada pela primeira vez, provavelmente sob certa tensão emocional por parte do paciente. Entre os que tiveram inicialmente valores abaixo de $140 \mathrm{~mm}$. Hg. para a sistólica é de $85 \mathrm{~mm}$. Hg. para a diastólica, verifícou 2,3\% de hipertensão após 10 anos, e 3,8\% após 20 anos. Entre os que na primeira visita tiveram valores entre 140 e 160 para a sistólica e 85 e 100 para a diastólica, 59,7\% de hipertensões após 10 anos e 82,5\% após 20 anos. Nessa estatística o A. considera como hipertensão valores superiores a 160-100, para sistólica e diastólica, respectivamente.

\section{BIBLIOGRAFIA}

1 - Alam, M. e Smirk, F. H. - Blood pressure raising reflexes in health, essential hypertension and renal hypertension. - Clin Sc. 3:259, 1938. (Resumo por Hines Jr. em A. H. J., 17:124, 1939).

2 - Ayman; D. e Goldshine, A. D. - Cold as a standard stimulus of blood pressure. - New England J. Med. 17 :650, 1938. (Resumo por Naide, em Am. Heart J., $17: 256,1939)$.

3 - Ayman, D. e Golds Hine, A. D. - Breath-holding test: simple standard stimulus of blood pressure. - Arch. Int. Med. 63:899-906, 1939. (Resumo in Year Book of General Medicine, 1939, pg. 496).

4 - BAK, M. - "Kalt-test" - Untersuchungen an Schwangeren, Gebärenden und Wöchnerinnen. - Monatschf. f. Geburtshilfe und Gynäk. $105: 24-29,1937$. 
5 - Briggs, J. F. e Oerting, H. - Vasomotor response of normal and hypertensive individuals to standard stimulus (cold). - Minnesota Med. $16: 481,1933(*)$.

6 - Briggs, J. F. e Oerting, H. - The prognostic value of the cold pressor test in pregnancy. - Minnesota Med. 20:382, 1937 (*).

7 - Chesley, L. e Chesley, E. - The cold pressor test in pregnancy. Surg, Gynec. and Obst. $69: 436-440,1939$.

8 - Craig, W. e Brown, G. - Unilateral and bilateral resection of the major and minor splanchnic nerves, its effects in cases of essential hypertension. - Arch. of Int. Med. 54:577-596, 1934.

9 - Crocr, C. - La prova "freddo-pressione" durante il ciclo menstruale. Monatschr. f. Geburtsh. u. Gynäk. 110:334-342, 1940.

10 - Dickmann, W. S. e Michel, H. L. - Thermal study of vasomotor lability in pregnancy. - Arch. Int. Med. 55:420, 1935.

11 - Dickmann, W. S., Michel, H. L. e Woodruff, P. W. - The cold pressor test in pregnancy. - Am. J. Obst. Gyn. 36:408-412, 1938.

12 - Hines JR., E. A. e Brown, G. E. - A standard stimulus for measuring vasomotor reactions: its application in the study of hypertension. Proc. Staff. Meet. Mayo Clinic 7:322, 1932 (*).

13 - Hines Jr., E. A. e Brown, G. E. - A standard test for measuring the variability of blood pressure; its significánce as an index of the pre-hypertensive state. - Ann. Int. Med., $7: 209-217,1933$ (*).

14 - Hines JR., E. A. e Brown, G. E. - The hereditary factor in the reaction of blood pressure to standard stimulus (cold). Preliminary report. Proc. Staff. Méet. Mayo Clin. $10: 371$-373, 1935.

15 - Hines Jr., E. A. e Brown, G. E. - The cold pressor test for measuring the reactibility of the blood pressure; data concernig 571 normal and hypertensive subjects. - Am. Heart J. 11:1-9, 1936:

16 - Hines JR., E. A. - Reaction of the blood pressure of 400 school children to standard stimulus. - J. Am. Med. Assoc. 108:1249-1250, 1937.

17 - Hrnes JR., E. A. - Hereditary factor in essential hyṕertension. - Ann. Int. Med. 11:593-601, 1937 (Resumo in Year Book of General Medicine, 1938, pg. 500).

18 - Hines JR., E. A. e Roth, G. M: - Effect of tobacco on blood pressure as measured by standard smoking test. - Proc. Staff. Meet. Mayo Clin. 13:524-526, 1938. (Resumo in Year Book of General Medicine, 1939, pag. 497).

19 - Hines Jr., E. A. - Technic of the Cold Pressor Test. - Proc. Staff. Meet. Mayo Clin. 14:185-187, 1939.

20 - Hines JR., E. A. - The significance of vascular hyperreaction as measured by the cold pressor test. - Collected Papers of The Mayo Clinic and The Mayo Foundation - 31:329-336, 1939.

21 - Hines JR., E. A. - Range of normal blood pressure and subsequente development of hypertension. - J. A. M. A., $115: 271-274,1940$.

22 - Miller, J. H. e Bruger, M. - The cold pressor reaction in normal subjects and in patients with primary (essential) and secondary (renal) hypertension. - Am. Heart J. 18:329-333, 1939.

23 - Randall, L. M., Murray, G. E. e Mussey, R. D. - The "cold test" in pregnancy. A preliminary report of its use in pre natal care. Am. J. Obst. Gyn. $29: 362-365,1935$.

24 - Pickering and Kissin - Effects of adrenalin and of cold on blood pressure in human hypertension. - Clin. Sc., $2: 201,1936\left(^{*}\right)$.

25 - ReID, D. E. e TeEL, H. M. - A' study of the "cold test" in normal and toxemic pregnancy. - Am. J. Obst. Gyn. $35: 305-309,1938$.

26 - Schwab, E., Curb, D., Mathews, L. e Schultze, V. E. - Blood pressure response to a standard stimulus in the white and negro races. - Proc. Soc. Exp. Biol. and. Med. 32:583-585, 1935.

$\left(^{*}\right)$ Trabalhos não consultados para elaboraçāo do presente artigo. 
27 - Schwab, E. e Curb, D. - A note on the diagnosis of hypertensive cardiovascular disease without hypertension. - The Journal of Lab. and Clin. Med., $24: 125-127,1938$.

28 - Thomas, C. B. e Warthin, T. A. - The response of normal dogs and dogs with experimental hypertension to a standard cold stimulus. - The Am. Heart J., $19: 316-329,1940$.

29 - Yates, M. R. e Wood JR., J. E. - Vasomotor response of non-hypertensive individuals to standard cold stimulus. - Proc. Soc. Exp. Biol. and Med., 34:560-562, 1936.

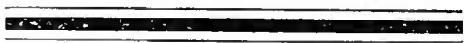

\section{Laboratório de Análises Clínicas} 照

\section{Dr. A. Rodrigues Metto \\ Dr. Roberto Franco do Amaral}

Rua Quintino Bocaiúvas - 122, 1.0 Tel.: 2-6565 


\section{PYORRHÉA}

Gengivas sangrentas, dentes abalados e mau halito: Resultados positivos em 8 dias, com o especifico PYORRHON.

CONSULTAS: $30 \$ 000$.

DEMONSTRAÇÕES PRATICAS AOS SENHORES MEDICOS E DENTISTAS.

\section{DR. CLINEO PAIM}

Rua Barão de Itapetininga, 120 $5 .^{\circ}$ andar Salas, 505 e 506 (Casa Guatapará)

Tel: 4-4050 SÃO PAULO

\section{ENDOSCOPIOS}

PARA TODOS OSCASOS DE

DIAGNOSTICA

E CIRURGIA

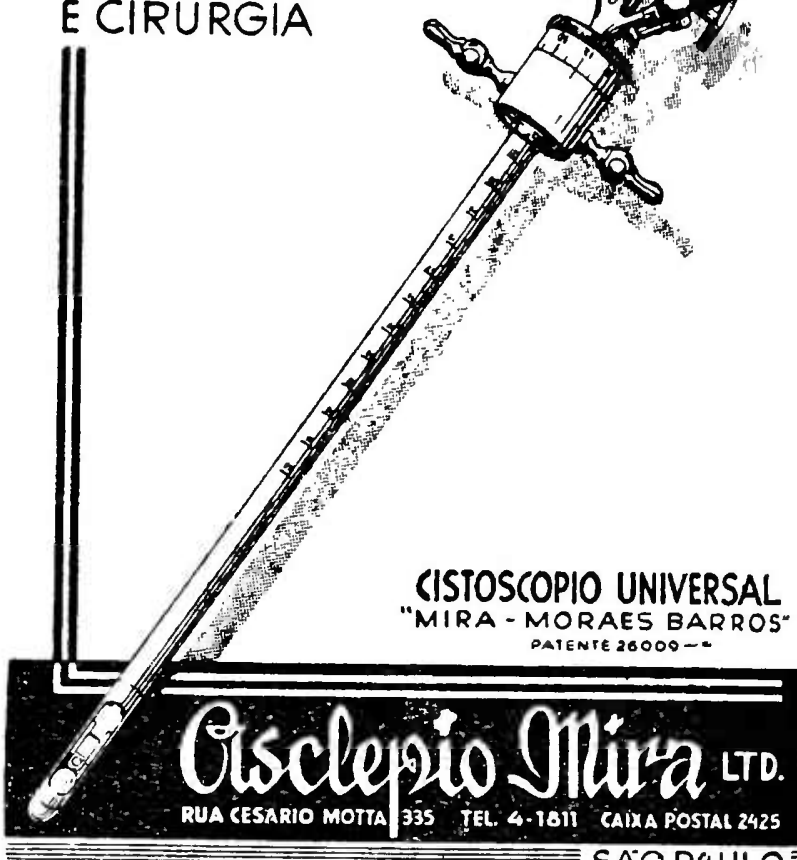

\section{Pyorrhon}

Um medicamento que veio. resolver os casos de Gengivites e Pyorhée

\section{A T E S T A D O}

E' para mim um prazer atestar que venho empregando em minha clinica com os mais brilhantes resultados, o Pyorrhon, medicamento de escol para o tratamento da Piorrhéa Alveolar e das Gengivites.

Tambem venho calorosamente recomendando o seu uso aos meus pacientes, porque assim fazendo estes teem assegurada a perfeita saude do seu meio bucal.

O Pyorrhon é um preparado que pela propaganda honesta com que é lançado e pelos seus meritos, merece da nossa classe a melhor acolhida.

São Paulo, 6 de Outubro de 1939.

Octavio Demacq Rosas.

Receite PYORRHON aos seus clientes 